THE LANCET, AUgust 27, 1853.

\section{Climital 王ectures}

\author{
ov
}

DISEASES AND INJURIES OF JOINTS.

\author{
By SAMUEL SOLLY, Esq., F.R.S. \\ SURGRON TO ST. THOMAS'S HOSPITAL.
}

GENTLemen,-From the diseases of the hip-joint we will descend to those of the knee, but you must take the metaphor in an anatomical, not a surgical sense; for whether we regard the importance of the joint in locomotion, its complicated anatomical machinery, its exposed position, both in relation to atmospheric changes and liability to injury from violence, it is a joint to be placed as high in the scale of practical interest as that which we have just been considering.

Strumous affections of the knee-joint are fearfully frequent in this country, but if the scrofulous diathesis is not very intense, you may generally succeed in arresting the destructive tendency of the disease, and procure an anchylosed joint, if you are consulted in an early stage, and your patient will conform to the rules laid down for his welfare.

You will all remember a girl I have just presented from Queen's ward, cured. It is true that she was in the hospital nearly four years; few therefore of the gentlemen attending this lecture will remember her appearance on admission. am quite sure that you would never recognise the present healthy, happy-looking young woman as the poor, wan, miserable, emaciated child that occupied the same bed four years ago. She was so ill that amputation of the limb was quite out of the question; she would have died on the operatingtable. I had, however, very little hope of being able to save life; but, thanks to the cod-liver oil, perfect and entire rest, good and nourishing diet, with repeated moxa over the joint, both her life and her limb have been saved.

It is not necessary for me to read to you the details of the case; the plan of treatment is comprehended in those few words, and its result has been for some time before your eyes.

Bat you must not suppose such simple measures will always be so successful, or such a course of treatment is unattended with anxiety; to be conducted safely it must be conducted with judgment. You must watch such cases very carefully day by day, lest they sink from the wasting discharge which ensues, or are destroyed by the constitutional irritation which they excite.

Cases occasionally occur in which there is no doubt that the life of the patient has been sacrificed in the attempt to save the $\lim b$. I had a case of this kind under my care in Henry's ward, eight years ago. The subject was a young man about twenty of age, with unequivocal strumous disease of the kneejoint; he was very much out of health when he came into the hospital, but without any of the physical signs of disease of the lungs. Considering it a fair case to try to save the joint, $I$ adopted such measures as have been already described, but he became worse, and died, after being in the house four months, with tubercular disease of the lungs. I do not mean to say that amputation would have prevented this fatal termination, but I confess that if $I$ had a similar case $I$ should recommend amputation at an earlier period. I do not think with some surgeons that the removal of a scrofulous joint increases the tendency to tuberculous disease of the lungs. I always consider it my duty to examine the chest carefully, and obtain the opinion of my medical colleague as to the condition of lungs before operating; but unless there is very positive evidence of disease of those organs, $I$ do not think it right to deprive my patient of this mode of relief.

In two cases $I$ have amputated scrofulous knee-joints where there were some symptoms of tubercular deposit in the lungs, but in both cases the patients recovered from the operation, regained their health, and I believe that they are alive now, six years having elapsed. As a rule I endeavour to procure anchylosis, and thus save the limb; if, however, I find the health of my patient does not improve with entire rest of the joint and the employment of those tonics which $I$ consider most indicated, then I lose no time in advising the operation. I must again repeat my conviction that of all the tonics we possess there are none equal to cod-liver oil in strumous cases, and that by the assistance of that medicine $I$ have been able to preserve limbs which I doubt if I could without it.

The next case that I shall relate to you illustrates the fatal termination, but $\mathrm{I}$ believe that this sad result was entirely occasioned by the patient's own imprudence.

No. 1565 .
"George M- aged thirty-one years, lighterman, was admitted into George's ward, under Mr. Solly, Dec. 8th, 1851, with ulceration of cartilages and synovitis of right knee-joint.

"History.-Appears to be of a healthy family: Had syphilis and gonorrhoea when he was about twenty-six years old, and took mercury in moderation. Never had rheumatism. He is married. He states that ten years ago he perceived a hard lump, like a nutshell, on the outside of the patella, between the femur and the tibia, which gradually extended like $\mathbf{a}$ cord in the shape of a horse-shoe over the condyles of the femur to the other side. At this time he began to feel pain; when he was working, the skin was not red, and there was no external inflammation."

The account which an illiterate patient gives of the rise and progress of his disease is never very clear or satisfactory; nevertheless, it is your duty to elicit as much as you can. The conclusion which I draw from this account is, that the disease commenced with chronic or subacuto inflammation, and thickening of the synovial membrane in the front of the joint.

"His knee did not disturb him at night. He cannot account for the origin of the disease, but says he may have fallen or knocked his knee in the barge. He went to a surgeon, who prescribed iodide of potassium ointment and rest; the ointment he used, but not the rest."

This poor fellow, in taking the medicine but rejecting the rest, was like a great many other patients, even among the higher clases, who ought to know better: he just adopted that portion of the prescription which suited his inclination. Many gentlemen come to your consulting-room in the morning to be relieved of symptoms which are solely the effect of their own indiscretion and intemperance in eating and drinking, and they expect you to cure them by physic, while they pursue the same inane course. They do not object to take medicine; will even consent, if the case requires it, to the application of leeches or blisters; but object decidedly to leaving off their wine; or, in the instance of a mercurial course, will not on any account confine themselves to the house. Now, gentlemen, when you meet with such patients, you must be decided, even at the risk of losing your patient. Such patients only do you more harm than good; you are better without them and their money, whatever it might amount to, if they will not adhere to those rules of diet, \&c., which are essential to their health.

"The cord-like swelling continued, and the pain became worse, occurring especially when he pulled on or took off his boots. He continued working, and has even walked twenty miles in a day. This state continued until five years ago, when he began to feel difficulty in flexing his leg. The angle of flexion he was able to perform gradually became less acute, and then more obtuse, until now the limb is nearly straight. Two years ago he went to Mr. Cock, of Guy's, having aggravated the pain by a fall, who prescribed rest and iodine ointment, leeches and poultices, and some stomachic medicine. He rested, however, very imperfectly, only on Sundays.

"Last May he came here to Mr. Solly; at this time he could scarcely bend his knee, and was incessantly knocking his toes against stones as he walked, and the capsule would fill now and then, and subside again. Mr. Solly ordered leeches, blisters, rest and quinine, and advised him to come into the hospital. However, he did not come in, but continued his employment until October, when he fell down the Waterman's Stairs, and somehow had his knee forcibly bent under him ; this gave him excruciating pain, but he hobbled home, and found the knee-joint immensely swollen. He poulticed and rested it, and the swelling went down, and he came. backwards and forwards in a cart as an out-patient of $\mathrm{Mr}$. Solly's, and was at last prevailed on to come in on the $8 \mathrm{th}$ of December.

"Dec. 8th.-The limb is now nearly straight and immovable, all motion giving great pain. When he fell down the Waterman's Stairs, the forcible flexion was accompanied by a sensation, he says, of the breaking up of cartilage. The affected leg is atrophied, and he has lost flesh generally. There are occasional severe aching and shooting pains, which sometimes awake him at night. There is great bulging of the capsule around the patella, and his general health is much impaired. He sweats a little at night, but eats well, and has a tolerably healthy complexion. He is losing weight. The pulse is good. The knee-joint measures round sixteen inches and a half. Put up in a M'Intire's splint, and to take iodine mixture, \&c."

Regarding this as a case of synovitis running on to ulceration 
of the cartilages, in which nature had already nearly effected anchylosis, I determined to try and assist her in her course. It was true that the poor fellow, in his anxiety to provide for his family by his daily labour, had done much to counteract the conservative process; still $I$ think it not at all improbable that this would have been effected if he had not had the misfortune to fall down the stairs, and rupture the adhesions which had been formed. But this was not all the mischief that this injury induced: it set up fresh inflammation of suppurative character, for which you will perceive the following treatment was adopted, with the view of subduing the inflammation:-

"Jan. 1st.-A large horse-shoe-shaped blister was ordered. The knee became gradually smaller, but he did not like the restraint of a splint, so the limb was taken out and placed on a pillow beneath a cradle, and the blister dressed with strong mercury ointment."

In the treatment of these affections of joints, where you are endeavouring to procure anchylosis as the only chance of saving both life and limb, you will frequently find your patient secretly as well as openly counteracting your efforts. I speak especially of hospital patients. More than once I have had patients complain of the restraint of the splint, and remove it themselves, not because they really suffered pain from the position or confinement of the limb, but because they were afraid of losing the use of the joint; and no words could persuade them that their safety alone depended on the possibility of producing a stiff joint. I have known this from the confession of the patients themselves. I mention it, not to induce you to shut your ears or disregard the complaints of patients,-on the contrary, I think that they always deserve attention,-but to warn you against deceit from this cause.

The joint measures fifteen inches and a half round; no pain.

"Feb. 1st.-The leg and thigh were encased with gutta percha, and rolled, the knee being covered with mercury ointment. Complains of loss of appetite; slightly salivated. " 8 th.-Mercury discontinued.

" 18 th.-Had a good night; feels better; pulse as yesterday.

"19th.-Tongue clean; passed a pretty good night; pulse frequent, small and soft; tongue moist and clean; appetite a little better.

"21st.-Opium at night; slept three hours; castor oil in the morning; operated freely.

"March 8th.-Fluctuation detected in knee and calf, evidently connected the one with the other. The splint was removed, and Mr. Solly made an opening in the calf, and about eight ounces of curdy pus escaped. The limb was rolled. Ordered roast meat daily, and porter; beef-tea, wine, arrowroot, eggs, mutton chop.

"9th.-Limb unrolled; about four ounces of fotid pus escaped; much constitutional disturbance; does not sleep well is thirsty and feverish. The knee measures less by an inch since the escape of the pus.

"10th.-Pus flows from the wound freely; general health as yesterday.

"13th.-Integument over knee puffy; there was a sense of fluctuation, and an incision was made below the outer condyle; no pus escaped; pus flows from calf."

I now felt that the only prospect of being able to save the life of my patient was to amputate the limb as soon as his general health and the state of the limb would permit the operation, and I therefore requested the opinion of my colleague, Mr. Green, on this matter. Considered there was too much inflammatory fever to operate then, but thought antputation must be the issue.

"Mr. Solly made an incision above the head of the filuia; a small quantity of pus escaped.

" 16 th.-Pus flows from the first and third wounds; sleeps badly; is feverish at night; has no appetite; tongue morbidly clean and red; skin dry; bowels confined.

" 17 th.-Passed a good night; had thirty minims of tincture of opium; feels better; pulse small, frequent, weak.

" 24 th.-Tincture of opium, forty minims. Did not sleep well; sweats; tongue moist; skin ditto; pulse frequent, but he has rather more power; three wounds discharging pus freely.-Two o'clock P.M.: Mr. Solly amputated the thigh about five inches above the knee, and making the posterior fiap, gave escape to a large quantity of pus."

It is a matter of so much importance to the man who has lost his natural leg to have a long lever to move the artificial substitute which art provides for him, that in an amputation of the thigh, you must not be induced to carry your incisions high up the thigh in order to avoid cutting into an abscess.
The presence of a portion of the wall of an abscess in the flap of the stump does not materially delay its healing.

" The femoral artery was compressed by Mr. Clark, and not above two or three ounces of blood were lost." You will perceive by this report that I was assisted by my able colleague Mr. Clark; and I assure you that such assistance is of the greatest value in conducting an amputation where it is so important not to lose a drop more blood than can be avoided.

In compressing the artery at the groin, the pressure is not applied until the moment before the incision is made, and there is, therefore, no turgescence of the veins of the limb as there is under the use of the tourniquet, the pressure of which accasions considerable loss of venous blood from the lower portion of the limb. "Chloroform was administered: the femoral vein was tied."

In some cases of amputation, the femoral vein bleeds so profusely that it is absolutely necessary to tie it. This profuse venous hæmorrhage generally occurs in feeble subjects long wasted by disease. The vein dilates under the upward column, the valves become useless, and the regurgitation is fearful. But do not think, gentlemen, that it is a matter of light importance whether the vein is tied or not. All the older surgeons of the present day held it to be a very dangerous practice; and I am convinced that that opinion was not lightly embraced, but was the result of observations on the serious mischief following this step. In modern times it has been the fashion to assert that there is no danger whatever in applying a ligature to a vein. My own opinion is, that both opinions are in the extreme. I can state from the experience of more than thirty years in this large hospital, that veins may be tied with impunity; but $I$ am also bound to add that $I$ have seen phlebitis follow the nse of the ligature, and a fatal result the sequence, if not the consequence of the practice. Indeed I believe that this very case is an illustration of the mischief which sometimes ensues from it.

It is quite true that we found sufficient visceral disease to account for this poor fellow's death, without being obliged to look at the state of the veins to explain it; and it is also true that phlebitis and pyemia occur in subjects when no vein has been ligatured, still it is my duty to call your attention to all the facts of the case, that they may be stored by you, and compared with the other facts you may be able to obtain on this subject during your sojourn in this borough of Southwark: and I must again repeat, that a ligature should not be put on a vein, unless the hremorrhage from that vein is so great as to endanger the safety of your patient.

Description of linee-joint, examined one hour after amputation, by Dr. Bristowe.-The knee-joint was entirely disorganized; the cartilages were completely removed from the surface of the patella. The surface exposed to the external condyle was quite denuded, and the exposed bone was nearly smooth and firm; the surface opposing internal condyle was more or less covered by a pulpy substance, but there was no cartilage. The articular surface of the femur was almost denuded; that over the external condyle completely so. The bone was compact everywhere. The surface opposed to the patella was nearly smooth, but irregular and eaten into hollows opposite the tibia. The internal condyle was also much exposed; small patches of pulpy substance adhered to it in places. The inner articular surface of the tibia was completely bare, and the bone roughened; the outer was almost covered entirely by a pulpy membrane. The inner inter-articular cartilage had disappeared; the outer was reduced to a thin rim. The crucial ligaments were softened and pulpy; the whole synovial membrane of the joint was inflamed and discoloured, and in places Sigerated, so as to communicate with the sinuses, some of whisie tonded upwards to the thigh, beneath and between the muscies o $\approx$ as almost completely to invest the thigh-bone; some extended downwards behind the muscles of the calf.

"Two hours after the operation, thirty minims of tincture of opium were administered, and not having the desired effect two hours afterwards, twenty-five minims more; he dozed a little about half-past twelve. He vomited, and did so three times between this and two o'clock. He dozed after this; had a restless night; pulse frequent, soft and full; tongue moist and clear; not much appetite, but still has taken wine; beef-tea and custard-pudding, \&c.

" $26 \mathrm{th}$.-Thirty minims of tincture of opium. Tongue clean and moist; pulse less frequent, soft, and more full; appetite better; a slight discharge of pus from stump.

" 2 th. - Had thirty minims of tincture of opium. Passed a better night. Took castor oil this morning; has operated freely; bowels have not been opened before for a week; tongue 
clean; skin moist; pulse frequent and feeble; appetite a little better (as yesterday;) wound looks healthy.

" 29 th. - Wound healthy; twenty minims of tincture of opium; did not sleep well; tongue and pulse good; thirsty however.

"30th.-Had thirty minims of tincture of opium, but did not sleep well. This morning his purgative operated very much, the fres being very offensive; tongue clean; pulse good.Half-past seven P.M.: He had a fit of shivering, and after that he became hot, and sweated a great deal; he vomited his food; he had thirty minims of tincture of opium, but passed a restless night. Took brandy, beef-tea, wine, \&c. Effervescent mixture, (soda water.)

"31st.-Nine A.M.: Looks haggard; skin cool and moist; tongue brown in the centre, white at edges; pulse without power, and frequent; no action in the stump beyond the discharge of a small quantity of very thin pus. Mr. Solly ordered him carbonate of ammonia, five grains ; tincture of cinchona, one drachm, in effervescent mixture, every six hours, and as much brandy and nourishment as he could take, but he can take no solid food.-Twelve A.M.: Mr. Green saw him; had rallied considerably. In the evening he became restless again, and sweated. Had thirty minims of tincture of opium at eleven o'clock; restless at night.-Four A.M.: In coughing up some mucus was sick.

"April 1st.-Nine A.Mr.: Mr. Solly dressed stump; no action in it; no granulations; no pus; pulse frequent; no power; tongue brown in the centre; had a slight rigor in the night; takes his liquid food; bowels slightly opened this morning.Seven P.M.: Anxions countenance; clammy skin; pulse faltering, and without power; takes little nourishment, and ate piece of a mutton-chop; dozed in the night; had no opium; had a fit of coughing about three o'clock, and was unable to spit up the mucus. Became cold, and died at four o'clock A.Mr., perfectly conscious of what was taking place.

The description which has been just given you of the state of the knee-joint after amputation is a faithful portrait of acute suppurative inflammation invading a joint already the seat of chronic disease.

"Post-mortem Examination.-Chest: Pleura on both sides universally adherent by old adhesions. Both lungs thickly studded from apex to base, and groups of small tubercles; intervening lung-structure congested in parts, but otherwise healthy, and free from any consolidation. None of the tubercles in a softened condition. Heart healthy. Abdomen: Peritonæum healthy; liver rather large, pale, and soft, slightly fatty. The right kidney contained a large mass of encysted tubercular matter at the upper portion of the pelvis, into which it projected; the structure otherwise appeared to be tolerably healthy. Left kidney apparently healthy. Stomach and intestines, spleen and pancreas, healthy. Stump: The femoral vein was filled with pus, which extended a short distance into the iliac vein, the middle and upper portions of which were filled with coagulum, and softened at the lower part. The extremity of the vein at the stump was patulous; the contained pus was thick and yellow in the lower part of the vein, and in the upper portion of a dirty-brown colour; the parietes of the vein were thickened throughout. Femoral artery contracted and healthy, and its extremity in the stump plugged with rather loosely-adherent coagulum, half an inch in length."

ON DISEASES OF THE SKIN,

IN REFERENCE TO THEIR CONSTITUTIONAL ORIGIN AND TREATMENT,

Bx THOMAS HUNT, Esq., F.R.C.S.

SURGEON TO THE WESTERN DISPENSARY FOR DISEASES OF THE SEIN.

THE phraseology of medical science has changed so much of late that the term constitutional, as applied to pathology, familiar as it has long been to the profession, may seem to require a definition. As applied to treatment its meaning is obvions enough, denoting some mode of relieving local disease other than the direct medication of the diseased structure; but a local disease can so seldom exist per se, independently of some lesion of the general system, that to argue in favour of the constitutional origin, or nature of any particular local affection, may seem superfluous. Strictly speaking, indeed, there is the same kind of relation between a pimple and the constitution, as between the leap of a grasshopper and the inertia of the earth: the insect kicks the earth from him as truly as he leaps away from the earth, although the one movement is sensible and appreciated, and the other only theoretical. If a child falls into a tub of hot water, the whole surface of the body is blistered. It is a local affection, but how soon do the symptoms show that it is one in which the whole system participates. A carbuncle, an eruption of small-pox, scarlatina, or measles, and an attack of erysipelas or pemphigus, are all so many illustrations of the fully admitted truth, that a severe affection of the skin, whether caused by accident or otherwise, involves the constitution in the general disturbance. In the milder forms of skin disease the general lesion may be less obvious, but, from analogy, we are bound to conclude that it exists. If a person is inveulated for small-pox, and but one pustule appears, that pustule is preceded and accompanied by some degree of pyrexia. Nor is it possible for a pimple to be thrown out spontaneously on the surface of the body without some previous lesion, however slight, either of the solids or the circulating fluids of the general system: else we should have an effect without a cause. In like manner, every cutaneous disease, whether arising spontaneously, like lepra or herpes, or whether resulting from contagion, as scabies or porrigo, either originally or ultimately involves the constitution, more or less obviously, in the changes which are talking place in the capillary system. As the brain takes cognizance of every disturbance in the extremities of the nerves, so the heart receives and reflects an impression when the minute vessels, however distant from the centre of circulation, become congested or inflamed. In fact, a sympathy exists throughout both systems and in all parts of the frame, so that every part of the body suffers with every member, and each member with the whole body, the local disease, when communicated from without, becoming the cause of the constitutional disturbance, and vice versà, the general cachexy, when it exists primarily, becoming, in its turn, the canse of the local affection.

I have been anxious to explain in the outset more fully than many readers may think it to have been necessary, the nature and necessity of this relation, because there are many intelligent practitioners who doubt and deny the universality of the connexion between the local and the general, and a still larger number who, admitting it as a general principle, practically ignore it in the treatment of certain forms of local disease. I have, indeed, long ago ventured to publish the opinion that the difficulties attending the treatment of chronic cutaneous diseases, are mainly attributable to neglecting that vigilant attention to the state of the general health which always suggests the most correct indications for treatment. Increasing familiarity with these diseases has abundantly confirmed me in these views, and the chief purport of this paper and others which may follow, is to demonstrate by facts, and illustrate by cases, some of the multiform varieties of disordered health, which oftentimes obscurely complicate, and not seldom originate, cutaneous disease.

The more obvious forms of deranged health which are found associated with eruptions, may be classed under the heads of gout, dyspepsia, visceral congestion, plethora, anæmia, neuralgia, scrofula, syphilis, and the like. But more frequently the general disorder is less obvious, though not always less important; and to these more obscure deviations from health, (to which the patient himself is often in a great degree a stranger,) it will be my special object to entreat attention.

There are few maladies which give more trouble or less satisfaction to the general practitioner than the various chronic forms of eruption which break out in schools or other large establishments, the inmates of which partake of the same diet, breathe the same atmosphere, sleep in the same dormitory, observe the same habits, and are engaged in the same pursuits. A large number of children, say ten or twenty out of every hundred, are often found affected with a similar eruption. By cleanliness and attention the disease will often yield to local treatment, but in a short time it returns. Sometimes it assumes the form of scabies, running rapidly into pustules, sometimes of scald-head or ringworm, sometimes of boils, whitlows, or blisters. A theorist, fresh from school, well grounded in rudimentary medical treatises, will tell me that $I$ am confounding together essentially different diseases; that scabies must be cured by sulphur, ringworm by detergents, boils by poultices, \&c.; but experienced practitioners know too well that a faithful and diligent use of these remedies, with due attention to cleanliness, will do little or nothing towards removing these diseases when they occur in schools or large domiciliary establishments. And the first thing to be done towards the treatment is to unlearn the Willanean nosology and diagnosis, to look beneath the surface, and to study the nature and causes of that vitiated condition of 